\title{
Noncommutative Dirac and Klein-Gordon oscillators in the background of cosmic string: spectrum and dynamics
}

\author{
Baloitcha Ezinvi, ${ }^{1, *}$ Mahouton Norbert Hounkonnou, ${ }^{1, \dagger}$ Emanonfi Elias N’Dolo,,${ }^{1} \ddagger$ and Dine Ousmane Samary ${ }^{2,1, \S}$ \\ ${ }^{1}$ Faculté des Sciences et Techniques, \\ International Chair in Mathematical Physics and Applications (ICMPA-UNESCO Chair), \\ University of Abomey-Calavi, 072B.P.50, Cotonou, Republic of Benin \\ ${ }^{2}$ Max Planck Institute for Gravitational Physics, \\ Albert Einstein Institute, Am Mühlenberg 1, 14476, Potsdam, Germany
}

(Dated: Tuesday $4^{\text {th }}$ September, 2018)

\begin{abstract}
From a study of an oscillator in a $4 D$ NC spacetime, we establish the Hamilton equations of motion. The formers are solved to give the oscillator position and momentum coordinates. These coordinates are used to build a metric similar to that describing a cosmic string. On this basis, Dirac and Klein-Gordon oscillators are investigated. Their spectrum and dynamics are analysed giving rise to novel interesting properties.
\end{abstract}

Key words: Noncommutative quantum and field theory, Dirac and Klein-Gordon oscillator, cosmic string.

PACS numbers: 03.65.Aa, 04.62.+v, 03.65.Ge

\section{INTRODUCTION}

Noncommutative (NC) field theory could play a special role in the description of particle physics near the Planck length $\lambda_{p}=\sqrt{G \hbar / c^{3}}$. This theory was the subject of intense study during the last three decades and provided a very interesting new class of quantum field theories with intriguing and sometimes unexpected features. The idea of noncommutativity of spacetime came from Snyder [1]. Its geometric analysis was given by Alain Connes [2]-[3]. The wide class of works on this subject and the physical implications such as the quantum Hall effect [4]-[5], the string theory static solutions [6], the matrix model or the $2 D$ quantum gravitation theory [7]-[9] opened new outlook on the study of physics. This made them particularly interesting and challenging for purposes of particle physics model building.

However, the NC spacetime generalizes the ordinary space by assuming the nonvanishing commutation relations between coordinates as $\left[\hat{x}^{\mu}, \hat{x}^{\nu}\right]=i \theta^{\mu \nu}$, where $\left(\theta^{\mu \nu}\right)$ is skew-symmetric constant tensor. The operator algebra of such NC spacetime can be represented by the algebra of functions when the ordinary multiplication of functions is replaced by the so-called Moyal star-product:

$$
\begin{aligned}
(f \star g)(x)= & \mathbf{m}\left[\exp \left[\theta^{\mu \nu} \partial_{\mu} \otimes \partial_{\nu}\right](f \otimes g)(x)\right], \\
& \mathbf{m}(f \otimes g)=f \cdot g, \quad f, g \in C^{\infty}\left(\mathbb{R}^{D}\right)
\end{aligned}
$$

(see [14] and references therein). Another noncommutativity is described when the momentum components become NC, i.e. $\left[\hat{p}_{\mu}, \hat{p}_{\nu}\right]=i \bar{\theta}_{\mu \nu}$, where $\left(\bar{\theta}_{\mu \nu}\right)$ is related to the magnetic field [10]. Several physical models were studied in this NC spacetime, such as the model of harmonic oscillator, the dynamics of the relativistic particles, and the scattering theory.

Recently, the Dirac and KG oscillators were studied in curve spacetime by introducing the tedrad fields $e_{a}^{\mu}$, or, equivalently, the metric $\mathrm{g}^{\mu \nu}=e_{a}^{\mu} e_{b}^{\nu} \eta^{a b}$, where $\eta$ is the flat spacetime metric [11]-[17]. These models were also implemented in the topologycal defect background metric [15]. An important question that we address here in this paper is the effects of noncommutativity on the dynamics of the spin orbit particle. We show that the noncommutativity of the spacetime transforms the Minkowski metric to the so-called cosmic string background. By giving the solution of the oscillator dynamics using the Hamilton equations of motion, we derive the corresponding deformation of the spacetime metric, which depends on the parameter $\theta$ and is similar to that describing a cosmic string. As application, we are interested in relativistic particles described by Dirac and Klein-Gordon (KG) oscillators. Several motivations lead to the study of these two models. See [11]-[24] for more details.

The paper is organized as follows. In the section (II), we provide the dynamics of a harmonic oscillator in NC spacetime. We show how this noncommutativity modifies the corresponding metric. In the section (III), we study the eigen-energies of the Dirac oscillator in the background of cosmic string. The same question is pointed out in the case of the KG oscillator. Section (IV) is devoted to concluding remarks.

\section{OSCILLATOR QUANTUM DYNAMICS ON NC SPACETIME}

*Electronic address: ezinvi.baloitcha@cipma.uac.bj

$\dagger$ Electronic address: norbert.hounkonnou@cipma.uac.bj

‡Electronic address: emanonfieliasndolo@yahoo.fr

$\S$ Electronic address: dine.ousmane.samary@aei.mpg.de
In this section, we study the quantum dynamics on $\mathrm{NC}$ spacetime. Using the Hamilton equation of motion of the coordinates system, we derive and solve the correspond- 
ing equations of motion. We show, by a novel approach, how a NC oscillator can be solved in the commutative spacetime, and how this may affect the spacetime geometric properties such as the metric tensor. Two cases of noncommutativity are considered. The case, when only coordinates are $\mathrm{NC}$, and the case where both coordinates and momentums are NC.

\section{A. Case of commutative momentum components}

We consider the spacetime geometry described with the NC coordinates $x^{\mu}$ and momentums $p_{\mu}, \mu=0,1,2,3$, which satisfy the star-commutation relations :

$$
\left[x^{\mu}, x^{\nu}\right]_{\star}=i \theta^{\mu \nu}, \quad\left[x^{\mu}, p_{\nu}\right]_{\star}=i \delta_{\nu}^{\mu}, \quad\left[p_{\mu}, p_{\nu}\right]_{\star}=0,
$$

in which, $\hbar:=1 ; \star$ denotes the Moyal star product. For $f, g \in C^{\infty}\left(\mathbb{R}^{4} \times \mathbb{R}^{4}\right)$ we write

$$
\begin{aligned}
f \star g & =\mathbf{m}\left[e^{\left(\mathcal{P}_{\theta}+\mathcal{P}_{\hbar}\right)}\right], \quad \mathbf{m}(f \otimes g)=f \cdot g \\
\mathcal{P}_{\theta} & =\frac{i \theta^{\mu \nu}}{2} \frac{\partial}{\partial x^{\mu}} \otimes \frac{\partial}{\partial x^{\nu}}, \\
\mathcal{P}_{\hbar} & =\frac{i}{2} \delta^{\mu \nu}\left(\frac{\partial}{\partial x^{\mu}} \otimes \frac{\partial}{\partial p^{\nu}}-\frac{\partial}{\partial p^{\mu}} \otimes \frac{\partial}{\partial x^{\nu}}\right) .
\end{aligned}
$$

The matrix $\theta^{\mu \nu}$ is chosen to be

$$
\theta^{\mu \nu}=\left(\begin{array}{cccc}
0 & \theta_{0} & \theta_{0} & \theta_{0} \\
-\theta_{0} & 0 & \theta & \theta \\
-\theta_{0} & -\theta & 0 & \theta \\
-\theta_{0} & -\theta & -\theta & 0
\end{array}\right), \quad \theta_{0}, \theta \in \mathbb{R}
$$

For instance, setting $\theta_{0}=0$ means that the time does not commute with the space coordinates and plays the role of evolution parameter. For all smooth function of coordinates and momentums $f(x, p)$, we have the following identities

$$
\begin{aligned}
& {\left[x^{\mu}, f(x, p)\right]_{\star}=i \theta^{\mu \beta} \frac{\partial f(x, p)}{\partial x^{\beta}}+i \delta^{\mu \beta} \frac{\partial f(x, p)}{\partial p^{\beta}},} \\
& {\left[p^{\mu}, f(x, p)\right]_{\star}=-i \delta^{\mu \beta} \frac{\partial f(x, p)}{\partial x^{\beta}} .}
\end{aligned}
$$

It is obvious that the $\mathrm{NC}$ coordinates are related to the commutative coordinates by the followings transformations:

$$
x^{\mu} \rightarrow x_{c}^{\mu}-\frac{\theta^{\mu \nu}}{2} p_{\nu, c}, \quad p_{\mu} \rightarrow p_{\mu, c},
$$

where the commutative variables satisfy the commutation relations $\left[x_{c}^{\mu}, x_{c}^{\nu}\right]_{\star}=0$ and $\left[x_{c}^{\mu}, p_{\nu, c}\right]_{\star}=i \delta_{\nu}^{\mu}$. Consider the Hamiltonian system, with Hamiltonian $H \in$ $C^{2}\left(\mathbb{R}^{8}, \mathbb{R}\right),(x, p) \in \mathbb{R}^{4} \times \mathbb{R}^{4}$. The Hamiltonian $H$ does not explicitly depend on the time $x_{c}^{0}$. Using the Taylor expansion we write:

$$
H(x, p)=\frac{|p|_{c}^{2}}{2}+V\left(x_{c}\right)+
$$

$$
\begin{aligned}
& \sum_{n=1}^{\infty} \frac{(-1)^{n}}{n !} \frac{\left(\theta p_{c}\right)^{j_{1}}}{2} \frac{\left(\theta p_{c}\right)^{j_{2}}}{2} \cdots \frac{\left(\theta p_{c}\right)^{j_{n}}}{2} \frac{d^{n} V\left(x_{c}\right)}{d x_{c}^{j_{1}} d x_{c}^{j_{2}} \cdots d x_{c}^{j_{n}}} \\
& \left(\theta p_{c}\right)^{j}=\theta^{j \ell} p_{\ell, c}, j, \ell=1,2,3 .
\end{aligned}
$$

The equations of the dynamics associated to the coordinates and momentums with the Hamiltonian (7) are

$$
\frac{d x_{c}^{\mu}}{d x_{c}^{0}}=i\left[x_{c}^{\mu}, H\right]_{\star}, \quad \frac{d p_{c}^{\mu}}{d x_{c}^{0}}=i\left[p_{c}^{\mu}, H\right]_{\star} .
$$

For any choice of potential $V(x)$, the above equations lead to a cumbersome system of nonlinear differential equations, not easily to solve. In the case where $V\left(x_{c}\right)$ is the harmonic oscillator potential, i.e. $V(x)=\frac{M\left|x_{c}\right|^{2}}{2}$, the Hamiltonian $H$ takes the form

$$
H=\frac{1}{2}\left|p_{c}\right|^{2}+\frac{M}{2}\left(\left|x_{c}\right|^{2}-\theta_{i j} x_{c}^{i} p_{c}^{j}+\frac{1}{4} \theta^{i j} \theta_{i k} p_{j, c} p_{c}^{k}\right) .
$$

We show in the sequel that the corresponding equations of motion can be solved in this particular case. These equations of motion are explicitly given by the following system:

$$
\left\{\begin{aligned}
\dot{x}_{c}^{1} & =-\frac{1}{2} M \theta x_{c}^{2}-\frac{1}{2} M \theta x_{c}^{3}-\left(1+\frac{M \theta^{2}}{2}\right) p_{c}^{1} \\
& -\frac{1}{4} M \theta^{2} p_{c}^{2}+\frac{1}{4} M \theta^{2} p_{c}^{3} \\
\dot{x}_{c}^{2} & =\frac{1}{2} M \theta x_{c}^{1}-\frac{1}{2} M \theta x_{c}^{3}-\left(1+\frac{M \theta^{2}}{2}\right) p_{c}^{2} \\
& -\frac{1}{4} M \theta^{2} p_{c}^{1}-\frac{1}{4} M \theta^{2} p_{c}^{3} \\
\dot{x}_{c}^{3} & =\frac{1}{2} M \theta x_{c}^{1}+\frac{1}{2} M \theta x_{c}^{2}-\left(1+\frac{M \theta^{2}}{2}\right) p_{c}^{3} \\
& +\frac{1}{4} M \theta^{2} p_{c}^{1}-\frac{1}{4} M \theta^{2} p_{c}^{2} \\
\dot{p}_{c}^{1} & =M x_{c}^{1}-\frac{1}{2} M \theta p_{c}^{2}-\frac{1}{2} M \theta p_{c}^{3} \\
\dot{p}_{c}^{2} & =M x_{c}^{2}+\frac{1}{2} M \theta p_{c}^{1}-\frac{1}{2} M \theta p_{c}^{3} \\
\dot{p}_{c}^{3} & =M x_{c}^{3}+\frac{1}{2} M \theta p_{c}^{1}+\frac{1}{2} M \theta p_{c}^{2}
\end{aligned}\right.
$$

where "dot" means the first order derivative with respect to the time $x_{c}^{0}:=t$. The system (10) can be solved, by using the expansion series method, to yield the general solutions:

$$
\begin{aligned}
& x_{c}^{j}(t)=\sum_{k=0}^{\infty}\left[a_{k}^{j}(\theta t)^{k} \cos (\sqrt{M} t)+b_{k}^{j}(\theta t)^{k} \sin (\sqrt{M} t)\right](11) \\
& p_{c}^{j}(t)=\sum_{k=0}^{\infty}\left[c_{k}^{j}(\theta t)^{k} \cos (\sqrt{M} t)+d_{k}^{j}(\theta t)^{k} \sin (\sqrt{M} t)\right](12)
\end{aligned}
$$

Note that $p_{c}^{j}=M \dot{x}_{c}^{j}$. Then the series $a_{k}^{j}$ and $b_{k}^{j}$ are related to $c_{k}^{j}$ and $d_{k}^{j}$ by the recursive relations

$$
\left\{\begin{array}{l}
c_{k}^{j}=(k+1) M \theta a_{k+1}^{j}+\sqrt{M^{3}} b_{k}^{j} \\
d_{k}^{j}=(k+1) M \theta b_{k+1}^{j}-\sqrt{M^{3}} a_{k}^{j}
\end{array} .\right.
$$

We consider the particular case in which we assume that there exist the constants $\alpha_{0}$ and $\beta_{0}$ such that

$$
\left\{\begin{array}{l}
\alpha_{0} b_{k}^{j}=(k+1) M \theta a_{k+1}^{j}+\sqrt{M^{3}} b_{k}^{j} \\
\beta_{0} a_{k}^{j}=(k+1) M \theta b_{k+1}^{j}-\sqrt{M^{3}} a_{k}^{j}
\end{array} .\right.
$$


Then, the series $a_{k}^{j}$ and $b_{k}^{j}$ satisfy the two-term recursive relations

$$
\begin{aligned}
& \left(\beta_{0}+\sqrt{M^{3}}\right) a_{k}^{j}-\frac{(k+1)(k+2) M^{2} \theta^{2}}{\alpha_{0}-\sqrt{M^{3}}} a_{k+2}^{j}=0 \\
& \left(\alpha_{0}-\sqrt{M^{3}}\right) b_{k}^{j}-\frac{(k+1)(k+2) M^{2} \theta^{2}}{\beta_{0}+\sqrt{M^{3}}} b_{k+2}^{j}=0
\end{aligned}
$$

the solutions of which are of the form:

$$
\begin{aligned}
& a_{k}^{j}=\Omega_{a}^{j} \frac{(-1)^{\frac{k}{2}}}{k !}\left(\frac{\left(\sqrt{M^{3}}-\alpha_{0}\right)\left(\sqrt{M^{3}}+\beta_{0}\right)}{M^{2} \theta^{2}}\right)^{\frac{k}{2}}, \\
& b_{k}^{j}=\Omega_{b}^{j} \frac{(-1)^{\frac{k}{2}}}{k !}\left(\frac{\left(\sqrt{M^{3}}-\alpha_{0}\right)\left(\sqrt{M^{3}}+\beta_{0}\right)}{M^{2} \theta^{2}}\right)^{\frac{k}{2}},
\end{aligned}
$$

where $\Omega_{a, b}^{j}$ are real constants. Letting $\eta^{2}=$ $\frac{\left(\sqrt{M^{3}}-\alpha_{0}\right)\left(\sqrt{M^{3}}+\beta_{0}\right)}{M^{2}}$ and $\alpha_{0}=\beta_{0}=\wp$ transforms the expressions (11) and (12) into the following form:

- For $\sqrt{M^{3}}>\wp$

$$
\begin{aligned}
x_{c}^{j}(t) & =\mathcal{R}_{e}\left[e^{i t \eta}\left(\Omega_{a}^{j} \cos \sqrt{M} t+\Omega_{b}^{j} \sin \sqrt{M} t\right)\right] \\
& =\cos (t \eta)\left(\Omega_{a}^{j} \cos \sqrt{M} t+\Omega_{b}^{j} \sin \sqrt{M} t\right) \\
p_{c}^{j}(t) & =\mathcal{R}_{e}\left[\wp e^{i t \eta}\left(\Omega_{a}^{j} \cos \sqrt{M} t+\Omega_{b}^{j} \sin \sqrt{M} t\right)\right] \\
& =\wp \cos (t \eta)\left(\Omega_{a}^{j} \cos \sqrt{M} t+\Omega_{b}^{j} \sin \sqrt{M} t\right) .
\end{aligned}
$$

- For $\sqrt{M^{3}}<\wp$

$$
\begin{aligned}
& x_{c}^{j}(t)=e^{-t \eta}\left(\Omega_{a}^{j} \cos \sqrt{M} t+\Omega_{b}^{j} \sin \sqrt{M} t\right) \\
& p_{c}^{j}(t)=\wp e^{-t \eta}\left(\Omega_{a}^{j} \cos \sqrt{M} t+\Omega_{b}^{j} \sin \sqrt{M} t\right) .
\end{aligned}
$$

These expressions represent the solution of the noncommutative oscillator in the commutative variables. They do not depend on the deformation parameter $\theta$, and hence are not affected by the noncommutativity of the spacetime.

Let us examine now how these solutions may modify the geometry. Let $\mathrm{g}_{c}$ and $\mathrm{g}$ are the metrics of ordinary and NC spacetime, respectively. We assume that $\mathrm{g}_{c, \mu \nu}:=$ $\eta_{\mu \nu}=\operatorname{diag}(-1,+1,+1,+1)$. Then using (6), (19) and (20) we get

$$
\begin{aligned}
\mathrm{g} & =\eta_{\mu \nu} d x^{\mu} d x^{\nu} \\
& =-d t^{2}+\sum_{j=1}^{3}\left(d x_{c}^{j} d x_{c}^{j}+\frac{\wp^{2} \theta^{j k} \theta^{j l}}{4} d x_{k, c} d x_{l, c}\right)
\end{aligned}
$$

which can explicitly be written in the matrix form as:

$$
\left(\mathrm{g}^{\mu \nu}\right)=\left(\begin{array}{cccc}
-1 & 0 & 0 & 0 \\
0 & 1+\frac{\wp^{2} \theta^{2}}{2} & \frac{\wp^{2} \theta^{2}}{4} & -\frac{\wp^{2} \theta^{2}}{4} \\
0 & \frac{\wp^{2} \theta^{2}}{4} & 1+\frac{\wp^{2} \theta^{2}}{2} & \frac{\wp^{2} \theta^{2}}{4} \\
0 & -\frac{\wp^{2} \theta^{2}}{4} & \frac{\wp^{2} \theta^{2}}{4} & 1+\frac{\wp^{2} \theta^{2}}{2}
\end{array}\right) .
$$

In the diagonal form we get

$$
\mathrm{g}_{d}=\operatorname{diag}\left(-1, \lambda_{1}^{2}, \lambda_{2}^{2}, \lambda_{3}^{2}\right),
$$

with eigenvalues

$$
\lambda_{1}^{2}=1, \quad \lambda_{2}^{2}=1+\frac{3 \theta^{2} \wp^{2}}{4}=\lambda_{3}^{2},
$$

and eigenvectors

$$
\begin{aligned}
& \mathbf{u}_{-1}=(1,0,0,0), \quad \mathbf{u}_{\lambda_{1}}=(0,1,-1,1), \\
& \mathbf{u}_{\lambda_{2}}=(0,-1,0,1), \quad \mathbf{u}_{\lambda_{3}}=(0,1,1,0) .
\end{aligned}
$$

Then the determinant of the metric $\mathrm{g}$, denoted by $g$, is $\operatorname{det}(\mathrm{g})=-\left(1+\frac{3 \theta^{2} \wp^{2}}{2}+\frac{9 \theta^{4} \wp^{4}}{16}\right)$. In a compact form, we get:

$$
\mathrm{g}=-d t^{2}+\sum_{j=1}^{3} a_{j}^{2}(\theta)\left(d x_{c}^{j}\right)^{2}, \quad a_{j}(\theta)=\lambda_{j},
$$

in which the parameters $a_{j}(\theta)=\lambda_{j}, j=1,2,3$ play the role of the scale factors.

\section{B. Case of noncommutative momentum components}

Here we consider the quantum spacetime described with the NC coordinates $x^{\mu}$ and momentums $p_{\mu}, \mu=$ $0,1,2,3$, which satisfy the star-commutation relations :

$$
\left[x^{\mu}, x^{\nu}\right]_{\star}=i \theta^{\mu \nu},\left[x^{\mu}, p_{\nu}\right]_{\star}=i \kappa_{\nu}^{\mu},\left[p_{\mu}, p_{\nu}\right]_{\star}=i \bar{\theta}_{\mu \nu},
$$

where the Moyal star product takes the form

$$
\begin{aligned}
f \star g & =\mathbf{m}\left[\exp \left(\mathcal{P}_{\theta}+\mathcal{P}_{\bar{\theta}}+\mathcal{P}_{\hbar}\right)\right] \\
\mathcal{P}_{\bar{\theta}} & =\frac{i \bar{\theta}^{\mu \nu}}{2} \frac{\partial}{\partial p^{\mu}} \otimes \frac{\partial}{\partial p^{\nu}} .
\end{aligned}
$$

The skew symmetric matrix $(\bar{\theta})$ is chosen to be

$$
\bar{\theta}^{\mu \nu}=\left(\begin{array}{cccc}
0 & \bar{\theta}_{0} & \bar{\theta}_{0} & \bar{\theta}_{0} \\
-\bar{\theta}_{0} & 0 & \bar{\theta} & \bar{\theta} \\
-\bar{\theta}_{0} & -\bar{\theta} & 0 & \bar{\theta} \\
-\bar{\theta}_{0} & -\bar{\theta} & -\bar{\theta} & 0
\end{array}\right), \quad \bar{\theta}_{0}, \bar{\theta} \in \mathbb{R}
$$

For instance, by choosing $\bar{\theta}_{0}=0$, the NC coordinates are related to the commutative coordinates by the relations:

$$
x^{\mu}=x_{c}^{\mu}-\frac{1}{2} \theta^{\mu \nu} p_{\nu, c}, \quad p^{\mu}=p_{c}^{\mu}+\frac{1}{2} \bar{\theta}^{\mu \nu} x_{\nu, c},
$$

such that the following commutation relations hold: $\left[x_{c}^{\mu}, x_{c}^{\nu}\right]_{\star}=0,\left[x_{c}^{\mu}, p_{\nu, c}\right]_{\star}=i \delta_{\nu}^{\mu},\left[p_{c}^{\mu}, p_{c}^{\nu}\right]_{\star}=0$. The tensor $\kappa$ takes the form:

$$
\kappa^{\mu \nu}=\left(1+\frac{\theta \bar{\theta}}{4}\right) \delta^{\mu \nu}
$$


Like (5) we get

$$
\begin{aligned}
& {\left[x^{\mu}, f(x, p)\right]_{\star}=i \theta^{\mu \beta} \frac{\partial f(x, p)}{\partial x^{\beta}}+i \delta^{\mu \beta} \frac{\partial f(x, p)}{\partial p^{\beta}},} \\
& {\left[p^{\mu}, f(x, p)\right]_{\star}=\frac{i \bar{\theta}^{\mu \beta}}{2} \frac{\partial f(x, p)}{\partial p^{\beta}}-i \delta^{\mu \beta} \frac{\partial f(x, p)}{\partial x^{\beta}} .}
\end{aligned}
$$

Then the Hamiltonian of the NC harmonic oscillator can be written as:

$$
\begin{aligned}
H & =\frac{1}{2}\left(\left|p_{c}\right|^{2}+\bar{\theta}_{i j} p_{c}^{i} x_{c}^{j}+\frac{1}{4} \bar{\theta}^{i j} \bar{\theta}_{i k} x_{j, c} x_{c}^{k}\right) \\
& +\frac{M}{2}\left(\left|x_{c}\right|^{2}-\theta_{i j} x_{c}^{i} p_{c}^{j}+\frac{1}{4} \theta^{i j} \theta_{i k} p_{j, c} p_{c}^{k}\right) .
\end{aligned}
$$

This leads to the following sytem of equations of motion

$$
\begin{cases}\dot{x}_{c}^{1} \quad=-\frac{1}{2}(M \theta+\bar{\theta}) x_{c}^{2}-\frac{1}{2}(M \theta+\bar{\theta}) x_{c}^{3} \\ & -\left(1+\frac{M \theta^{2}}{2}\right) p_{c}^{1}-\frac{1}{4} M \theta^{2} p_{c}^{2}+\frac{1}{4} M \theta^{2} p_{c}^{3} \\ \dot{x}_{c}^{2} & =\frac{1}{2}(M \theta+\bar{\theta}) x_{c}^{1}-\frac{1}{2}(M \theta+\bar{\theta}) x_{c}^{3} \\ & -\left(1+\frac{M \theta^{2}}{2}\right) p_{c}^{2}-\frac{1}{4} M \theta^{2} p_{c}^{1}-\frac{1}{4} M \theta^{2} p_{c}^{3} \\ \dot{x}_{c}^{3} \quad & =\frac{1}{2}(M \theta+\bar{\theta}) x_{c}^{1}+\frac{1}{2}(M \theta+\bar{\theta}) x_{c}^{2} \\ & -\left(1+\frac{M \theta^{2}}{2}\right) p_{c}^{3}+\frac{1}{4} M \theta^{2} p_{c}^{1}-\frac{1}{4} M \theta^{2} p_{c}^{2} \\ \dot{p}_{c}^{1} \quad & =-\frac{1}{2}(M \theta+\bar{\theta}) p_{c}^{2}-\frac{1}{2}(M \theta+\bar{\theta}) p_{c}^{3} \\ & +\left(M+\frac{\bar{\theta}^{2}}{2}\right) x_{c}^{1}+\frac{1}{4} \bar{\theta}^{2} x_{c}^{2}-\frac{1}{4} \bar{\theta}^{2} x_{c}^{3} \\ \dot{p}_{c}^{2} & =\frac{1}{2}(M \theta+\bar{\theta}) p_{c}^{1}-\frac{1}{2}(M \theta+\bar{\theta}) p_{c}^{3} \\ & +\left(M+\frac{\bar{\theta}^{2}}{2}\right) x_{c}^{2}+\frac{1}{4} \bar{\theta}^{2} x_{c}^{1}+\frac{1}{4} \bar{\theta}^{2} x_{c}^{3} \\ \dot{p}_{c}^{3} & =\frac{1}{2}(M \theta+\bar{\theta}) p_{c}^{1}+\frac{1}{2}(M \theta+\bar{\theta}) p_{c}^{2} \\ & +\left(M+\frac{\bar{\theta}^{2}}{2}\right) x_{c}^{2}-\frac{1}{4} \bar{\theta}^{2} x_{c}^{1}+\frac{1}{4} \bar{\theta}^{2} x_{c}^{2}\end{cases}
$$

After some algebra, and exploiting the expansion series method, we obtain:

$$
\begin{aligned}
x_{c}^{j}(t)= & \sum_{k=0}^{\infty} \sum_{\ell=0}^{\infty}(\theta t+\bar{\theta} t)^{k+\ell}\left[a_{k \ell}^{j} \cos (\sqrt{M} t)\right. \\
& \left.+b_{k \ell}^{j} \sin (\sqrt{M} t)\right] \\
p_{c}^{j}(t)= & \sum_{k=0}^{\infty} \sum_{\ell=0}^{\infty}(\theta t+\bar{\theta} t)^{k+\ell}\left[c_{k \ell}^{j} \cos (\sqrt{M} t)\right. \\
& \left.+d_{k \ell}^{j} \sin (\sqrt{M} t)\right] .
\end{aligned}
$$

Now by setting $p_{c}^{j}(t)=M \dot{x}_{c}^{j}(t)$, we get the following recursive relations:

$c_{k l}^{j}=\frac{M(\theta+\bar{\theta})(k+\ell+1)}{2}\left(a_{k+1, \ell}^{j}+a_{k, \ell+1}^{j}\right)+\sqrt{M^{3}} b_{k \ell}^{j}$

$d_{k l}^{j}=\frac{M(\theta+\bar{\theta})(k+\ell+1)}{2}\left(b_{k+1, \ell}^{j}+b_{k, \ell+1}^{j}\right)-\sqrt{M^{3}} a_{k \ell}^{j}$

$\alpha_{0}$ and $\beta_{0}$ are two constants such that $c_{k \ell}^{j}=\alpha_{0} b_{k \ell}^{j}$ and $d_{k \ell}^{j}=\beta_{0} a_{k \ell}^{j}$. We come to

$$
\begin{aligned}
\left(\beta_{0}+\sqrt{M^{3}}\right) a_{k \ell}^{j} & =\frac{M^{2}(\theta+\bar{\theta})^{2}}{4\left(\alpha_{0}-\sqrt{M^{3}}\right)}(k+\ell+1)(k+\ell+2) \\
& \times\left(a_{k+2, \ell}^{j}+2 a_{k+1, \ell+1}^{j}+a_{k, \ell+2}^{j}\right)
\end{aligned}
$$

and

$$
\left(\alpha_{0}-\sqrt{M^{3}}\right) b_{k \ell}^{j}=\frac{M^{2}(\theta+\bar{\theta})^{2}}{4\left(\beta_{0}+\sqrt{M^{3}}\right)}(k+\ell+1)(k+\ell+2)
$$

$$
\times\left(b_{k+2, \ell}^{j}+2 b_{k+1, \ell+1}^{j}+b_{k, \ell+2}^{j}\right) .
$$

The recursive relations (39) and (40) can be solved by setting $a_{k \ell}^{j}=a_{k+\ell}^{j}$ and $b_{k \ell}^{j}=b_{k+\ell}^{j}$. We then get the solutions:

$$
b_{k}^{j}=a_{k}^{j}=\Omega_{b, a}^{j} \frac{(-1)^{\frac{k}{2}}}{k !}\left(\frac{\left(\sqrt{M^{3}}-\alpha_{0}\right)\left(\sqrt{M^{3}}+\beta_{0}\right)}{M^{2}(\theta+\bar{\theta})^{2}}\right)^{\frac{k}{2}}
$$

The $x_{c}^{j}(t)$ and $p_{c}^{j}(t)$ are given by (19),(20),(21) and (22).

In the sequel, we perform some illustrations.

\section{APPLICATIONS TO THE RELATIVISTIC PARTICLES}

\section{A. The Dirac Oscillator}

We derive equation governing the Dirac oscillator in noncommutative space in the background of cosmic string. The model is described in the cylinder coordinates with the FLRW metric

$$
d s^{2}=-d t^{2}+\lambda_{1}^{2}(t) d r^{2}+\lambda_{2}^{2}(t) \alpha^{2} r^{2} d \varphi^{2}+\lambda_{3}^{2}(t) d z^{2},
$$

$-\infty<(t, z)<\infty, r \geq 0$ and $0 \leq \varphi \leq 2 \pi$. The parameter $\alpha$ is related to the linear mass density $\tilde{M}$ of the string by $\alpha=1-4 \tilde{M}$ and belongs to the interval $(0,1]$, corresponding to a deficit angle $\gamma=2 \pi(1-\alpha)$. We choose the scale factors $\lambda_{1}(t), \lambda_{2}(t)$ and $\lambda_{3}(t)$ to be now functions of time and implicitly on $\theta$. The particular case where these three parameters $\lambda_{j}$ are constant depending on $\theta$ (see (28)) will be discussed hereafter. In accordance with the metric (42) the tetrad $e_{a}^{\mu}(x)$ such that $\mathrm{g}^{\mu \nu}=e_{a}^{\mu} e_{b}^{\nu} \eta^{a b}$ is chosen to be

$$
\left[e_{a}^{\mu}\right]=\left(\begin{array}{cccc}
1 & 0 & 0 & 0 \\
0 & \frac{\cos \varphi}{\lambda_{1}} & \frac{\sin \varphi}{\lambda_{1}} & 0 \\
0 & -\frac{\sin \varphi}{\lambda_{2} \alpha r} & \frac{\cos \varphi}{\lambda_{2} \alpha r} & 0 \\
0 & 0 & 0 & \frac{1}{\lambda_{3}}
\end{array}\right)
$$

where the Greek indices is related to the curve space indices and the Latin indices to the Minkowski space indices. Remark that the tetrad (43) is not uniquely defined. Any tetrad is related to (43) by the local Lorentz transformation $\Lambda_{b}^{a}$ as $e_{a}^{\mu}(x)=\Lambda_{a}^{b}(x) e_{b}^{\mu}(x)$. The spinor connection is defined by

$$
\begin{aligned}
\Gamma_{\mu} & =-\frac{1}{8} \omega_{\mu}^{c d}\left[\gamma_{c}, \gamma_{d}\right] \\
\omega_{\mu}^{a b} & =e_{\nu}^{a} \Gamma_{\mu \sigma}^{\nu} e_{c}^{\sigma} \eta^{b c}-\eta^{b c} e_{c}^{\nu} \partial_{\mu} e_{\nu}^{a}
\end{aligned}
$$

$\gamma^{a}$ are the Dirac matrices in Minkowski space and $\Gamma_{\mu \sigma}^{\nu}$ is the Christoffel symbol. We also use the following notations related to the curve cylindrical coordinates: $(\mu, \nu)=(t, r, \varphi, z)$ and $(a, b)=(0,1,2,3)$ for 
the Minkowki space. Using (43) we can show that $\Gamma_{\mu}=\left(0, \Gamma_{r}, \Gamma_{\varphi}, \Gamma_{z}\right)$, where

$$
\begin{aligned}
\Gamma_{r}= & -\frac{\dot{\lambda}_{1}}{2} \gamma_{0} \gamma_{1} \cos \varphi-\frac{\dot{\lambda}_{1}}{2} \gamma_{0} \gamma_{2} \sin \varphi \\
\Gamma_{\varphi}= & \frac{\dot{\lambda}_{2}}{2} \alpha r \gamma_{0} \gamma_{1} \sin \varphi-\frac{\dot{\lambda}_{2}}{2} \alpha r \gamma_{0} \gamma_{2} \cos \varphi \\
& -\frac{1}{2}\left(1-\frac{\lambda_{2}}{\lambda_{1}} \alpha\right) \gamma_{1} \gamma_{2}, \\
\Gamma_{z}= & -\frac{\dot{\lambda}_{3}}{2} \gamma_{0} \gamma_{3} .
\end{aligned}
$$

In the above relation $\dot{\lambda}_{j}=\frac{d \lambda}{d t}$. The Dirac matices are $\gamma^{\mu}=e_{\ell}^{\mu} \gamma^{\ell}$, explicitly writen as:

$$
\begin{aligned}
\gamma^{t} & =\gamma^{0} \\
\gamma^{r} & =\frac{\gamma^{1}}{\lambda_{1}} \cos \varphi+\frac{\gamma^{2}}{\lambda_{1}} \sin \varphi \\
\gamma^{z} & =\frac{\gamma^{3}}{\lambda_{3}}, \\
\gamma^{\varphi} & =-\frac{\gamma^{1}}{\lambda_{2} \alpha r} \sin \varphi+\frac{\gamma^{2}}{\lambda_{2} \alpha r} \cos \varphi
\end{aligned}
$$

where we take the standard Dirac matrix to be

$$
\gamma^{0}=\left(\begin{array}{cc}
1 & 0 \\
0 & -1
\end{array}\right), \gamma^{i}=\left(\begin{array}{cc}
0 & \sigma^{i} \\
-\sigma^{i} & 0
\end{array}\right), i=1,2,3 .
$$

$\sigma^{i}$ are the Pauli matrices. The Dirac equation is the Euler-Lagrange equation of motion of the action

$$
S[\psi, \bar{\psi}, \Gamma]=\int \sqrt{-g} d^{4} x \bar{\psi} \hat{\mathcal{M}} \psi
$$

where $g=\operatorname{det} \mathrm{g}^{\mu \nu}=-\lambda_{1}^{2} \lambda_{2}^{2} \lambda_{3}^{2} \alpha^{2} r^{2}$ and

$$
\hat{\mathcal{M}}=i \gamma^{\mu}\left(\nabla_{\mu}+\Gamma_{\mu}\right)-i \gamma^{r} \gamma^{0} M \omega r-M
$$

We get

$$
\left[i \gamma^{\mu}\left(\nabla_{\mu}+\Gamma_{\mu}\right)-i \gamma^{r} \gamma^{0} M \omega r-M\right] \psi=0 .
$$

$M$ is a mass of the Dirac particle, $\psi$ is a spinor fourcomponents of the wave function; $\nabla_{\mu}$ is

$$
\nabla_{\mu}=h_{\mu}^{-1} \frac{\partial}{\partial x^{\mu}}, \quad \mathrm{g}^{\mu \nu}=h_{\mu}^{-2} \eta^{\mu \nu}
$$

In general $\nabla_{\mu}$ given in (51) is not a Hermitian operator and its components do not commute. So, the following definition will be used:

$$
\nabla_{\mu}(\cdot)=|g|^{-1 / 4} \partial_{\mu}\left(|g|^{1 / 4} \cdot\right)
$$

such that, for $\mathrm{g}^{\mu \nu}$ defined in (42) we find:

$$
\begin{aligned}
\nabla_{0} & =\frac{1}{2}\left(\frac{\dot{\lambda}_{1}}{\lambda_{1}}+\frac{\dot{\lambda}_{2}}{\lambda_{2}}+\frac{\dot{\lambda}_{3}}{\lambda_{3}}\right)+\frac{\partial}{\partial t} \\
\nabla_{r} & =\frac{1}{2 r}+\frac{\partial}{\partial r} \\
\nabla_{\varphi} & =\frac{\partial}{\partial \varphi} \\
\nabla_{z} & =\frac{\partial}{\partial z}
\end{aligned}
$$

These expressions mean that the wave equation $\varepsilon_{n}^{0} \psi=$ $i \partial_{t} \psi$ is modified as

$$
\varepsilon_{n} \psi=i \nabla_{0} \psi=i\left[\frac{1}{2}\left(\frac{\dot{\lambda}_{1}}{\lambda_{1}}+\frac{\dot{\lambda}_{2}}{\lambda_{2}}+\frac{\dot{\lambda}_{3}}{\lambda_{3}}\right)+\frac{\partial}{\partial t}\right] \psi
$$

In the case where $\lambda_{j}, j=1,2,3$, are such that $\dot{\lambda}_{j} / \lambda_{j}$ are constants, the energy spectrum takes the form

$$
\varepsilon_{n}=\varepsilon_{n}^{0}+\frac{i}{2}\left(\frac{\dot{\lambda}_{1}}{\lambda_{1}}+\frac{\dot{\lambda}_{2}}{\lambda_{2}}+\frac{\dot{\lambda}_{3}}{\lambda_{3}}\right)=\varepsilon_{n}^{0}+\varepsilon_{n}^{\lambda}
$$

Now let us assume that $\lambda_{j}, j=1,2,3$ are the constants given in (26), and the particle moves in $(x, y)$ plane. Then, $\varepsilon_{n}=\varepsilon_{n}^{0}$ and $\varepsilon_{n}^{0}$ need to be computed. We use the variables separation method in the Dirac equation (50) as follows:

$$
\psi(t, r, \varphi, z)=e^{-i \varepsilon_{n}^{0} t}\left(\begin{array}{c}
\widetilde{\psi}_{a}(r, \varphi) \\
\widetilde{\psi}_{b}(r, \varphi)
\end{array}\right)
$$

The $z$ dependence of the wave function is removed due to the phase factor of the form $e^{i k z}$, in which $k$ may be vanished. Using (59) we come to the two following differential equations:

$$
\begin{aligned}
& i \lambda_{1}\left(\sigma^{1} \cos \varphi+\sigma^{2} \sin \varphi\right)\left(M-\varepsilon_{n}^{0}\right) \widetilde{\psi}_{A} \\
& +\left[\frac{\partial}{\partial r}+M \omega r-\left(\frac{\lambda_{1}}{2 \alpha \lambda_{2}}-1\right) \frac{1}{r}+\frac{\lambda_{1}}{\lambda_{2}} \frac{i \sigma^{3}}{\alpha r} \frac{\partial}{\partial \varphi}\right] \widetilde{\psi}_{B}=0 \\
& i \lambda_{1}\left(\sigma^{1} \cos \varphi+\sigma^{2} \sin \varphi\right)\left(M+\varepsilon_{n}^{0}\right) \widetilde{\psi}_{B} \\
& -\left[\frac{\partial}{\partial r}-M \omega r-\left(\frac{\lambda_{1}}{2 \alpha \lambda_{2}}-1\right) \frac{1}{r}+\frac{\lambda_{1}}{\lambda_{2}} \frac{i \sigma^{3}}{\alpha r} \frac{\partial}{\partial \varphi}\right] \widetilde{\psi}_{A}=0
\end{aligned}
$$

Let us define the differential operators:

$$
\begin{aligned}
H_{1} & =\left[\frac{d}{d r}+M \omega r+\frac{\lambda_{1}}{\lambda_{2} r}\left(\frac{1}{2 \alpha}+\frac{m \sigma^{3}}{\alpha}+\frac{\lambda_{2}}{\lambda_{1}}\right)\right] \\
& \times\left[\frac{d}{d r}-M \omega r-\frac{\lambda_{1}}{\lambda_{2} r}\left(\frac{1}{2 \alpha}+\frac{m \sigma^{3}}{\alpha}-\frac{\lambda_{2}}{\lambda_{1}}\right)\right] \\
H_{2} & =\left[\frac{d}{d r}+M \omega r-\frac{\lambda_{1}}{\lambda_{2} r}\left(\frac{1}{2 \alpha}+\frac{m \sigma^{3}}{\alpha}-\frac{\lambda_{2}}{\lambda_{1}}\right)\right] \\
& \times\left[\frac{d}{d r}-M \omega r+\frac{\lambda_{1}}{\lambda_{2} r}\left(\frac{1}{2 \alpha}+\frac{m \sigma^{3}}{\alpha}+\frac{\lambda_{2}}{\lambda_{1}}\right)\right] .
\end{aligned}
$$

By setting

$$
\begin{aligned}
& \varepsilon_{a}=\lambda_{1}\left(M-\varepsilon_{n}^{0}\right), \quad \varepsilon_{b}=\lambda_{1}\left(M+\varepsilon_{n}^{0}\right), \\
& \widetilde{\psi}_{a}(r, \varphi)=e^{i m \varphi} \widetilde{\psi}_{a}(r), \quad \widetilde{\psi}_{b}(r, \varphi)=e^{i m \varphi} \widetilde{\psi}_{b}(r)
\end{aligned}
$$

we arrive at the eigenvalue problems

$$
H_{1} \widetilde{\psi}_{a}=\varepsilon_{a} \varepsilon_{b} \widetilde{\psi}_{a}, \quad H_{2} \widetilde{\psi}_{b}=\varepsilon_{a} \varepsilon_{b} \widetilde{\psi}_{b}
$$

Now let us recast the spinors $\widetilde{\psi}_{a}$ and $\widetilde{\psi}_{b}$ as

$$
\widetilde{\psi}_{a}=\left(\begin{array}{c}
\widetilde{\psi}_{a 1} \\
\widetilde{\psi}_{a 2}
\end{array}\right), \quad \widetilde{\psi}_{b}=\left(\begin{array}{c}
\widetilde{\psi}_{b 1} \\
\widetilde{\psi}_{b 2}
\end{array}\right)
$$


The functions $\widetilde{\psi}_{a i}, i=1,2$ and $\widetilde{\psi}_{b i}, i=1,2$ satisfy the well known Laguere polynomial equation:

$$
\frac{d^{2} \widetilde{\psi}_{l}}{d r^{2}}+\frac{2}{r} \frac{d \widetilde{\psi}_{l}}{d r}+\left(\frac{\eta_{2}}{r^{2}}-M^{2} \omega^{2} r^{2}+\eta_{0}\right) \widetilde{\psi}_{l}(r)=0,
$$

where $l=a 1, a 2$ or $b 1, b 2$, and $\eta_{0}, \eta_{2}$ are two constants depending on $l$, given by

$$
\begin{aligned}
& \left\{\begin{array}{l}
\eta_{0}=-\varepsilon_{a} \varepsilon_{b}-M \omega\left(1+\frac{1+2 m}{\alpha} \frac{\lambda_{1}}{\lambda_{2}}\right) \\
\eta_{2}=-\frac{\lambda_{1}^{2}}{\lambda_{2}^{2}} \frac{1+2 m}{2 \alpha}\left(\frac{1+2 m}{2 \alpha}-\frac{\lambda_{2}}{\lambda_{1}}\right)
\end{array} \quad l=a 1\right. \\
& \left\{\begin{array}{l}
\eta_{0}=-\varepsilon_{a} \varepsilon_{b}-M \omega\left(1+\frac{1-2 m}{\alpha} \frac{\lambda_{1}}{\lambda_{2}}\right) \\
\eta_{2}=-\frac{\lambda_{1}^{2}}{\lambda_{2}^{2}} \frac{1-2 m}{2 \alpha}\left(\frac{1-2 m}{2 \alpha}-\frac{\lambda_{2}}{\lambda_{1}}\right)
\end{array} \quad l=a 2\right. \\
& \left\{\begin{array}{l}
\eta_{0}=-\varepsilon_{a} \varepsilon_{b}+M \omega\left(1+\frac{1+2 m}{\alpha} \frac{\lambda_{1}}{\lambda_{2}}\right) \\
\eta_{2}=-\frac{\lambda_{1}^{2}}{\lambda_{2}^{2}} \frac{1+2 m}{2 \alpha}\left(\frac{1+2 m}{2 \alpha}-\frac{\lambda_{2}}{\lambda_{1}}\right)
\end{array} \quad l=b 1\right. \\
& \left\{\begin{array}{l}
\eta_{0}=-\varepsilon_{a} \varepsilon_{b}+M \omega\left(1+\frac{1-2 m}{\alpha} \frac{\lambda_{1}}{\lambda_{2}}\right) \\
\eta_{2}=-\frac{\lambda_{1}^{2}}{\lambda_{2}^{2}} \frac{1-2 m}{2 \alpha}\left(\frac{1-2 m}{2 \alpha}-\frac{\lambda_{2}}{\lambda_{1}}\right)
\end{array} \quad l=b 2\right.
\end{aligned}
$$

The general solution of equation (68) is given by

$$
\widetilde{\psi}_{l}(r)=\sqrt{\frac{(M \omega)^{\lambda+\frac{1}{2}}}{\pi \Omega(\lambda)}} e^{-\frac{1}{2} M \omega r^{2}} r^{\lambda-\frac{1}{2}} L_{n}^{\lambda}\left(M \omega r^{2}\right)
$$

where

$$
\begin{aligned}
& \lambda=\frac{1}{2} \sqrt{1-4 \eta_{2}}, \\
& n=\frac{\eta_{0}-2 M \omega-M \omega \sqrt{1-4 \eta_{2}}}{4 M \omega} \\
& \Omega(\lambda)=\int_{0}^{\infty} e^{-z} z^{\lambda+\beta} L_{n}^{\lambda}(z)^{2} d z,
\end{aligned}
$$

The eigenvalues $\varepsilon_{n}^{0}$ giving the energies of the model become

$$
\begin{aligned}
& \text { - for }|m| \leq \frac{1}{2}\left(\frac{\lambda_{2}}{\lambda_{1}} \alpha-1\right) \\
& \left\{\begin{array}{l}
\varepsilon_{n}^{0}=\left[M^{2}+\frac{4 M \omega}{\lambda_{1}^{2}}(n+1)\right]^{\frac{1}{2}} \\
\varepsilon_{n}^{0}=\left[M^{2}+\frac{2 M \omega}{\lambda_{1}^{2}}\left(2 n+1-\frac{1 \pm 2 m}{\alpha} \frac{\lambda_{1}}{\lambda_{2}}\right)\right]^{\frac{1}{2}},
\end{array}\right.
\end{aligned}
$$

- for $|m| \geq \frac{1}{2}\left(\frac{\lambda_{2}}{\lambda_{1}} \alpha-1\right)$

$$
\left\{\begin{array}{l}
\varepsilon_{n}^{0}=\left[M^{2}+\frac{2 M \omega}{\lambda_{1}^{2}}\left(2 n+1+\frac{1 \pm 2 m}{\alpha} \frac{\lambda_{1}}{\lambda_{2}}\right)\right]^{\frac{1}{2}} \\
\varepsilon_{n}^{0}=\left[M^{2}+\frac{4 M \omega n}{\lambda_{1}^{2}}\right]^{\frac{1}{2}}
\end{array} .\right.
$$

Remark 1. Our analysis highlights a new degeneracy of the energy spectrum for the different values of the parameter $m$. This result sheds light on the fact that the spectrum (77) does not exist in the literature and should be considered as a new feature caused by the bounds of $m$. We have also shown that $\epsilon_{n}^{0}$ depends on the deformation parameter $\theta$ or $\lambda_{j}$. We write $\varepsilon_{n}^{0}=\varepsilon_{n}^{0}(\theta)$. In the commutative limit, in which $\lambda_{j}=1$, or $\theta=0$, the expression (77) is not well defined. In this situation the energy spectrum is given in (78). This corresponds to the results computed in [11]-[18] (and references therein). Note that the same analysis can be made in the case of non vanishing electromagnetic fields solved in the commutative case in [13].

\section{B. The Klein-Gordon oscillator}

This section aims at applying the method used in the section (II) to the KG equation. Consider a scalar field $\Phi$ written in the cylindrical coordinates as $\Phi=\Phi(t, r, \varphi, z)$. The KG oscillator is given by the following equation, (see [22] for more details):

$$
\left(\square_{\mathrm{g}}-M^{2}\right) \Phi=0,
$$

where the Dalembertian operator in the spacetime defined with the metric (42) is

$$
\square_{\mathrm{g}}=\left(\nabla_{\mu}+\Gamma_{\mu}+M \omega r_{\mu}\right)\left(\nabla^{\mu}+\Gamma^{\mu}-M \omega r^{\mu}\right) .
$$

The Einstein summation is applied in the cylindrical coordiantes, with $\mu=(t, r, \varphi, z)$ and $r_{\mu}=(0, r, 0,0)$.

Suppose that the eigenvalues $\lambda_{j}, j=1,2,3$ defined in (42) are constants as expected in (26). By replacing the relations (46) and (53) in the KG equation (79) we come to the differential equation

$$
\left[\frac{\partial^{2}}{\partial t^{2}}+O(r, \varphi, z)+M^{2}\right] \Phi=0
$$

where the operator $O(r, \varphi, z)$ is

$$
\begin{aligned}
O(r, \varphi, z)= & -\frac{1}{\lambda_{3}^{2}} \frac{\partial^{2}}{\partial z^{2}}-\frac{1}{\lambda_{1}^{2}}\left(\frac{1}{2 r}+\frac{\partial}{\partial r}\right)^{2} \\
& -\frac{1}{\lambda_{2}^{2} \alpha^{2} r^{2}}\left[\frac{\partial}{\partial \varphi} \pm \frac{i}{2}\left(1-\frac{\lambda_{2}}{\lambda_{1}} \alpha\right)\right]^{2} \\
& +\frac{3}{2 \lambda_{1}^{2}} M \omega+\frac{M^{2} \omega^{2}}{\lambda_{1}^{2}} r^{2}
\end{aligned}
$$

Equation (81) admits the variable separation as $\Phi(t, r, \varphi, z)=e^{-i \varepsilon_{n}^{0} t+i k z+i m \varphi} \tilde{\Phi}(r)$, in which the radial function $\tilde{\Phi}(r)$ satisfies the following equation:

$$
\left\{\frac{d^{2}}{d r^{2}}+\frac{1}{r} \frac{d}{d r}+\frac{\tilde{\eta}_{2}}{r^{2}}-M^{2} \omega^{2} r^{2}+\tilde{\eta}_{0}\right\} \tilde{\Phi}=0,
$$

where

$$
\begin{aligned}
& \tilde{\eta}_{2}=\left[\frac{1}{4}+\frac{\lambda_{1}^{2}}{\lambda_{2}^{2} \alpha^{2}}\left(m \pm \frac{1}{2}\left(1-\frac{\lambda_{2}}{\lambda_{1}} \alpha\right)\right)^{2}\right] \\
& \tilde{\eta}_{0}=-\frac{3}{2} M \omega-\lambda_{1}^{2}\left(M^{2}-\varepsilon_{n}^{0}{ }^{2}\right) .
\end{aligned}
$$

The solution of this equation is given in (73). The energy spectrum becomes

$$
\varepsilon_{n}= \pm\left\{M^{2}+\frac{1}{\lambda_{1}^{2}}\left[4 n+\frac{3}{2} M \omega+2+2 \sqrt{\tilde{\eta}_{2}}\right\}^{\frac{1}{2}} .\right.
$$


The commutative limit corresponding to $\lambda_{j}=1, j=$ $1,2,3$ is readily obtained.

\section{CONCLUDING REMARKS}

In this paper we have investigated the dynamics of the harmonic oscillator in NC spacetime. The differential equation of motion deduced from this analysis has been solved. The corresponding deformation of the spacetime metric has been given. As application, the Dirac and KG oscillators have been explicitly described in the background of cosmic string. We have proved that the Dirac oscillator exibits a new degeneracy of the energy spectrum, unknown in the literature. The case of time dependent eigenvalues $\lambda_{j}(t)$ may be examined in the core of forthcoming investigations.

\section{ACKNOWLEDGMENTS}

EB, MNH and EEN works are partially supported by the Abdus Salam International Centre for Theoretical Physics (ICTP, Trieste, Italy) through the Office of External Activities (OEA)-Prj-15. The ICMPA is in partnership with the Daniel Iagolnitzer Foundation (DIF), France. DOS research at Max-Planck Institute is supported by the Alexander von Humboldt Foundation.

\section{Appendix}

Proof. of relation (11) and (12)

Consider the equation of motion (10), rewriten as:

$$
\frac{d \mathbf{X}}{d t}=U \mathbf{X}
$$

where $\mathbf{X}=\left(x_{c}^{1}, x_{c}^{2}, x_{c}^{3}, p_{c}^{1}, p_{c}^{2}, p_{c}^{3}\right)$ and $U$ is a matrix:

$$
\left(\begin{array}{cccccc}
0 & -\frac{M \theta}{2} & -\frac{M \theta}{2} & -\left(1+\frac{M \theta^{2}}{2}\right) & -\frac{M \theta^{2}}{4} & \frac{M \theta^{2}}{4} \\
\frac{M \theta}{2} & 0 & -\frac{M \theta}{2} & -\frac{M \theta^{2}}{4} & -\left(1+\frac{M \theta^{2}}{2}\right) & -\frac{M \theta^{2}}{4} \\
\frac{M \theta}{2} & \frac{M \theta}{2} & 0 & \frac{M \theta^{2}}{4} & -\frac{M \theta^{2}}{4} & -\left(1+\frac{M \theta^{2}}{4}\right) \\
M & 0 & 0 & 0 & -\frac{M \theta}{2} & -\frac{M \theta}{2} \\
0 & M & 0 & \frac{M \theta}{2} & 0 & -\frac{M \theta}{2} \\
0 & 0 & M & \frac{M \theta}{2} & \frac{M \theta}{2} & 0
\end{array}\right),
$$

with characteristic polynomial

$$
P(\lambda, \theta)=\left(\lambda^{2}+M\right)\left(\lambda^{4}+M^{2}+\lambda^{2} M\left(3 \theta^{2} M+2\right)\right)
$$

The eigenvalues and the eigenvectors of the above matrix can be simply given. Then the exact solution of the equation (10) can be given analytically. Because of the size of the solutions which can be found with a computer software, we give here an alternative way to approximate the result as follows :
- All the eigenvalues can be separated into $\lambda_{+}^{(\ell)}$ and $\lambda_{-}^{(\ell)}, \ell=1,2,3$, such that

$$
\lambda_{ \pm}^{(\ell)}= \pm i \sqrt{M} \pm u^{\ell}(\theta, M)
$$

where $u^{\ell}(\theta, t)$ are functions depending on $\theta$ and $M$. More precisely, the first order computation gives:

$$
\begin{aligned}
& \lambda_{ \pm}^{(1)}= \pm i \sqrt{M} \\
& \lambda_{ \pm}^{(2)}= \pm i \sqrt{M} \pm i \frac{M \theta \sqrt{3}}{2}+O\left(\theta^{2}\right), \\
& \lambda_{ \pm}^{(3)}= \pm i \sqrt{M} \mp i \frac{M \theta \sqrt{3}}{2}+O\left(\theta^{2}\right),
\end{aligned}
$$

corresponding to eigenvectors

$$
\begin{gathered}
\mathbf{v}_{\mathbf{1}}=\left(\frac{i}{\sqrt{M}},-\frac{i}{\sqrt{M}}, \frac{i}{\sqrt{M}}, 1,-1,1\right) \\
\mathbf{v}_{\mathbf{2}}=\left(-\frac{i}{\sqrt{M}}, \frac{i}{\sqrt{M}},-\frac{i}{\sqrt{M}}, 1,-1,1\right) \\
\mathbf{v}_{\mathbf{3}}=\left(-\frac{\sqrt{M}}{2 M}(\sqrt{3}+i),-\frac{\sqrt{M}}{2 M}(\sqrt{3}-i), \frac{i}{\sqrt{M}},\right. \\
\left.\frac{1}{2}(i \sqrt{3}-1), \frac{1}{2}(i \sqrt{3}+1), 1\right)+\mathbf{O}\left(\theta^{2}\right)
\end{gathered}
$$

$$
\begin{aligned}
\mathbf{v}_{\mathbf{4}}= & \left(-\frac{\sqrt{M}}{2 M}(\sqrt{3}-i),-\frac{\sqrt{M}}{2 M}(\sqrt{3}+i),-\frac{i}{\sqrt{M}},\right. \\
& \left.-\frac{1}{2}(i \sqrt{3}+1),-\frac{1}{2}(i \sqrt{3}-1), 1\right)+\mathbf{O}\left(\theta^{2}\right)
\end{aligned}
$$

$$
\begin{aligned}
\mathbf{v}_{\mathbf{5}}= & \left(\frac{\sqrt{M}}{2 M}(\sqrt{3}-i), \frac{\sqrt{M}}{2 M}(\sqrt{3}+i), \frac{i}{\sqrt{M}},\right. \\
& \left.-\frac{1}{2}(i \sqrt{3}+1),-\frac{1}{2}(i \sqrt{3}-1), 1\right)+\mathbf{O}\left(\theta^{2}\right) \\
\mathbf{v}_{\mathbf{6}}= & \left(\frac{-\sqrt{M}}{2 M}(\sqrt{3}+i), \frac{\sqrt{M}}{2 M}(\sqrt{3}-i),-\frac{i}{\sqrt{M}},(10), \mathbf{O}\left(\theta^{2}\right)\right. \\
& \left.\frac{1}{2}(i \sqrt{3}-1), \frac{1}{2}(i \sqrt{3}+1), 1\right)
\end{aligned}
$$

The solution of the system (10) at the first order in $\theta$ can simply be written as:

$x_{c}^{j}=\left(a_{1}^{j}+\theta t a_{2}^{j}\right) \cos \sqrt{M} t+\left(b_{1}^{j}+\theta t b_{2}^{j}\right) \sin \sqrt{M} t(102)$ $p_{c}^{j}=\left(c_{1}^{j}+\theta t c_{2}^{j}\right) \cos \sqrt{M} t+\left(d_{1}^{j}+\theta t d_{2}^{j}\right) \sin \sqrt{M} t(103)$

where $a_{1,2}^{j}, b_{1,2}^{j}, c_{1,2}^{j}$ and $d_{1,2}^{j}$ are real constants, which depend on the mass parameter $M$. The same procedure can be extended to higher order terms of $\theta$ as follows: 
- Suppose that the eigenvalue $\lambda$ are reduced to (89), (90) and (91), i.e. $u^{\ell}(\theta, M) \equiv C^{\ell} \theta$.

$$
C^{\ell}=\left\{\begin{array}{cc}
0, & \ell=0 \\
\pm \frac{i M \sqrt{3}}{2}, & \ell=1 \\
\mp \frac{i M \sqrt{3}}{2}, & \ell=2
\end{array}\right.
$$

The solution of (86) becomes

$$
\begin{aligned}
X^{j} & =\sum_{ \pm} \sum_{\ell} r_{\ell}^{j} e^{ \pm u^{\ell}(\theta, M) t} e^{ \pm i \sqrt{M} t}, \\
& =\sum_{ \pm} \sum_{\ell} r_{\ell}^{j} e^{ \pm C^{\ell} \theta t} e^{ \pm i \sqrt{M} t}
\end{aligned}
$$

The constants $r_{\ell}^{j} \in \mathbb{R}$ are the components of the vectors $\mathbf{v}_{\mathbf{j}}, j=1,2, \cdots, 6$, and depend on $\theta$ and $M$. Setting $A^{j}=r_{\ell}^{j}\left(e^{C^{\ell} \theta t}+e^{-C^{\ell} \theta t}\right)$ and $B^{j}=$ $i r_{\ell}^{j}\left(e^{C^{\ell} \theta t}-e^{-C^{\ell} \theta t}\right)$ yields

$$
X^{j}=\left[A^{j} \cos (\sqrt{M} t)+B^{j} \sin (\sqrt{M} t)\right]
$$

Using the Taylor expansion of the quantities $A^{j}$ and $B^{j}$, the solutions (11) and (12) are well satisfied.
[1] H. S. Snyder, "Quantized space-time," Phys. Rev. 71, 38 (1947).

[2] A. Connes, "Noncommutative Geometry," ISBN9780121858605.

[3] A. Connes and J. Lott, Nucl. Phys. Proc. Suppl. 18B, 29 (1991).

[4] B. Harms and O. Micu, J. Phys. A 40, 10337 (2007) [hep-th/0610081].

[5] F. G. Scholtz, B. Chakraborty, S. Gangopadhyay and J. Govaerts, J. Phys. A 38, 9849 (2005) [condmat/0509331 [cond-mat.mes-hall]].

[6] N. Seiberg and E. Witten, JHEP 9909, 032 (1999) [hepth/9908142].

[7] J. Zinn-Justin, J. Statist. Phys. 157, 990 (2014) doi:10.1007/s10955-014-1103-y [arXiv:1410.1635 [math$\mathrm{ph}]$.

[8] J. Ambjrn, S. Khachatryan and A. Sedrakyan, Phys. Rev. D 92, no. 2, $026002 \quad$ (2015) doi:10.1103/PhysRevD.92.026002 [arXiv:1407.0076 [hep-th]].

[9] E. R. Livine, Class. Quant. Grav. 26, 195014 (2009) doi:10.1088/0264-9381/26/19/195014 [arXiv:0811.1462 [gr-qc]].

[10] L. Gouba, Int. J. Mod. Phys. A 31, no. 19, $1630025 \quad$ (2016) doi:10.1142/S0217751X16300258 [arXiv:1603.07176 [hep-th]].

[11] D. Ito, K. Mori, and E. Carrieri, Nuovo Cimento A 51, 1119 (1967).

[12] M. Moshinsky and A. Szczepaniak, J. Phys. A: Math. Gen. 22 (1989).

[13] F. M. Andrade and E. O. Silva, Eur. Phys. J. C 74, no. 12,3187 (2014) doi:10.1140/epjc/s10052-014-3187-6 [arXiv:1403.4113 [hep-th]].

[14] R. J. Szabo, Phys. Rept. 378, 207 (2003) doi:10.1016/S0370-1573(03)00059-0 [hep-th/0109162].

[15] J. Carvalho, C. Furtado and F. Moraes, Phys. Rev. A 84, no. 3, 032109 (2011). doi:10.1103/PhysRevA.84.032109

[16] M. S. Maior de Sousa, R. F. Ribeiro and E. R. Bezerra de Mello, Phys. Rev. D 93, no. 4, 043545 (2016) doi:10.1103/PhysRevD.93.043545 [arXiv:1511.02745 [hep-th]].

[17] H. Akcay and R. Sever, Eur. Phys. J. Plus 131, no. 7, 225 (2016). doi:10.1140/epjp/i2016-16225-1

[18] A. Boumali and H. Hassanabadi, Can. J. Phys. 93, no. 5, 542 (2015). doi:10.1139/cjp-2014-0276

[19] H. Hassanabadi, S. S. Hosseini and S. Zarrinkamar, Chin. Phys. C 38, 063104 (2014). doi:10.1088/1674$1137 / 38 / 6 / 063104$

[20] B. P. Mandal and S. K. Rai, Phys. Lett. A 376, 2467 (2012) doi:10.1016/j.physleta.2012.07.001 [arXiv:1203.2714 [hep-th]].

[21] V. I. Kukulin, G. Loyola and M. Moshinsky, In *College Park 1991, Hadron'91* 293-301

[22] M. Mohadesi and B. Mirza, Commun. Theor. Phys. 42, 664 (2004) [hep-th/0412122].

[23] E. O. Silva, S. C. Ulhoa, F. M. Andrade, C. Filgueiras and R. G. G. Amorim, Annals Phys. 362, 739 (2015). doi:10.1016/j.aop.2015.09.011

[24] B. Malekolkalami, K. Atazadeh and B. Vakili, Phys. Lett. B 739, 400 (2014) doi:10.1016/j.physletb.2014.11.003 [arXiv:1411.3623 [gr-qc]]. 